\title{
Buscando Soluções Sustentáveis para Áreas Úmidas Savânicas Brasileiras via Uso Múltiplo
}

\author{
Eliana Paixão ${ }^{1}$, Erica Cezarine de Arruda ${ }^{2}$, Wolfgang J. Junk ${ }^{3} \&$ Cátia Nunes da Cunha ${ }^{4}$
}

Recebido em 30/01/2018 - Aceito em 21/02/2019

\begin{abstract}
RESUMO - As áreas úmidas savânicas são responsáveis por múltiplos serviços e produtos ambientais; portanto, seu uso múltiplo deve ser incentivado. No Brasil, as áreas úmidas savânicas se encontram no Pantanal, no rio Araguaia (Ilha do Bananal), no rio Guaporé e nos lavrados de Roraima. Como signatário da Convenção Ramsar, o Brasil deve compatibilizar suas políticas públicas, através do reconhecimento dos múltiplos valores das áreas úmidas, alcançando, assim, o uso racional dos seus recursos e contribuindo para o desenvolvimento sustentável. No Brasil, áreas úmidas savânicas não são contempladas na legislação específica, e a orientação de seu manejo - uma vez reconhecida como área de uso restrito - deverá atender aos princípios das Convenções Ramsar e da Diversidade Biológica. Diante das demandas por produtos florestais madeireiros e não madeireiros, a adoção de planos de manejo diferenciados é necessária. Essa abordagem propõe formas de manejo ecologicamente benéficas, incorporando valores adicionais ao uso da terra e às formações florestais. $\mathrm{O}$ uso múltiplo em áreas úmidas é o tipo de manejo integrado e planejado em que os vários usos dos recursos devem estar interligados, tornando-se uma estratégia possível para prolongar a disponibilidade dos recursos e o uso viável da terra, e manter as características ecológicas desses ambientes. As principais vantagens do manejo de uso múltiplo são o uso diversificado de recursos naturais, o aumento do rendimento de produtos e serviços, a participação das partes interessadas na obtenção de benefícios de recursos naturais, a diminuição dos conflitos sociais e degradação dos recursos florestais, o incentivo à manutenção de áreas florestais e a proteção da sua biodiversidade, entre outros. O conhecimento sobre as áreas úmidas savânicas e seu uso potencial devem ser divulgados e incentivados, para que seu uso seja realizado de forma sustentável, garantindo seu aproveitamento por futuras gerações.
\end{abstract}

Palavras-chave: Manejo sustentável de áreas úmidas; savanas brasileiras; sustentabilidade; uso racional.

ABSTRACT - Seeking Sustainable Solutions for Brazilian Savannah Wetlands Via Multiple Use. Multiple use as a sustainable solution for wetland savannas. The wide-ranging environmental services and products provided by wetland savannas encourage their multiple use. In Brazil, wetland savannas are

\footnotetext{
Afiliação

Instituto Nacional de Ciência e Tecnologia em Áreas Úmidas/INCT-INAU, Programa de Pós-graduação em Ecologia e Conservação da Biodiversidade/PPG - ECB, Universidade Federal de Mato Grosso/UFMT, Av. Fernando Corrêa da Costa, $n^{\circ}$ 2367, Boa Esperança, Cuiabá/MT, Brasil. CEP: 78.060-900.

2 Instituto Nacional de Ciência e Tecnologia em Áreas Úmidas/INCT-INAU, Universidade de Cuiabá/UNIC, Av. Manoel José de Arruda, n 3.100, Jardim Europa, Cuiabá/MT, Brasil. CEP: 78.065-900.

3 Instituto Nacional de Ciência e Tecnologia em Áreas Úmidas/INCT-INAU, Av. Fernando Corrêa da Costa, n 2367, Boa Esperança, Cuiabá/MT, Brasil. CEP: 78.060-900.

4 Universidade Federal de Mato Grosso/UFMT, Instituto de Biociências/IB, Departamento de Botânica e Ecologia, Instituto Nacional de Ciência e Tecnologia em Áreas Úmidas/INCT-INAU. Av. Fernando Corrêa da Costa, n 2367, Boa Esperança, Cuiabá/MT, Brasil. CEP: 78.060-900.

E-mail

elianapaixao2@gmail.com,ericacezarine@yahoo.com.br,wjj@evolbio.mpg.de,biocnc@gmail.com
} 
found in the Pantanal, adjacent to the Araguaia River (Bananal Island) and Guaporé River, as well as in Roraima's lavrados. As a signatory to the Ramsar Convention, Brazil is obligated to fulfill its commitments to this treaty and thus formulate public policies that recognize the multiple values of wetlands. This combined approach will achieve the wise use of wetland resources as well as the sustainable development of the country's wetlands. However, in Brazil, wetland savanna management policies are lacking that recognizes the need to restrict the use of these areas, comply with the principles of the Ramsar Convention, and seek to maintain biological diversity. Given the intense demand for the timber and non-timber forest products, differentiated, ecologically beneficial forms of management that incorporate the added value of land and forest use should be adopted. The multiple, sustainable use of wetlands requires an integrated, planned approach to management in which the various uses of resources are interlinked in a viable strategy that ensures longterm resource availability, viable land use, and the maintenance of the ecological characteristics of these environments. The main advantages of a multiple-use strategy are: the diversified use of natural resources, increased yields of products and services, the involvement of stakeholders in obtaining benefits from natural resources, reduction of social conflicts as well as the degradation of forest resources, and encouraging the maintenance of forested areas and the protection of their biodiversity. It is also important to make knowledge about wetland savannas and their potential resources widely available, so that the sustainable use of these areas, now and by future generations, is guaranteed.

Keywords: Sustainable management of wetlands; brazilian savannas; sustainability; wise use.

RESUMEN - Buscando soluciones sostenibles para los humedales de la sabana brasileña a través del uso múltiple. Buscando soluciones sostenibles para los humedales sabanicos brasileñas via uso múltiple. Los humedales desempeñan múltiples servicios y productos ambientales, por lo que su uso múltiple debe ser incentivado. En Brasil, los humedales se encuentran en el Pantanal, río Araguaia (Isla del Bananal), río Guaporé y en los lavrados de Roraima. Brasil, como parte de la Convención Ramsar, debe compatibilizar sus políticas públicas, a través del reconocimiento de los múltiples valores de los humedales, consiguiendo alcanzar el uso racional de sus recursos y contribuyendo al desenvolvimiento sustentable. Los humedales en Brasil no presentan una legislación específica y ningún plan de manejo reconocida como área de uso restringido, teniendo en cuenta los principios de las Convenciones Ramsar y de la Diversidad Biológica. Frente a las demandas por productos forestales madereros y no madereros es necesaria la elaboración de planes de manejo diferenciados para los humedales. Este enfoque propone formas de manejo ecológicamente beneficiosas, incorporando valores adicionales al uso de la tierra y las formaciones forestales. El uso múltiple en los humedales es un tipo de manejo integrado y planificado, donde los diversos usos de estos recursos, deben estar interconectados, transformándose en una estrategia factible para prolongar la disponibilidad de los recursos, donde el uso viable de la tierra es mantener las características ecológicas de esos ambientes. Las principales ventajas del manejo de uso múltiple son: uso diversificado de recursos naturales, aumento del rendimiento de productos y servicios, participación de las partes interesadas en la obtención de beneficios de recursos naturales, disminución de los conflictos sociales y degradación de los recursos forestales, el incentivo al mantenimiento de áreas forestales y a la protección de su biodiversidad, entre otros. El conocimiento sobre los humedales sabanicos y su uso potencial, deben ser divulgados e incentivados para que su uso sea realizado de forma sustentable garantizando su aprovechamiento por las futuras generaciones.

Palabras clave: Manejo sostenible de los humedales; sabanas brasileñas; sustentabilidad; uso racional.

\section{Introdução}

$\mathrm{Na}$ interface entre os ecossistemas terrestre e aquático, ocorrem áreas periodicamente alagadas, denominadas áreas de transição aquático-terrestre (ATTZ) (Junk et al. 1989), que constituem a maior parte das áreas úmidas (AUs) brasileiras. No sistema Brasileiro de classificação de AUs proposto por Junk et al. (2014a), estas podem ser divididas em dois grupos: as AUs com nível de água relativamente estável e as AUs com nível de água flutuante (sistema de pulso de inundação). No Brasil, mais de $90 \%$ das AUs são sujeitas a diferentes tipos de pulso de inundação (Junk et al. 2014a, 2014b). As grandes paisagens de AUs brasileiras, as planícies de inundação, são produtos de uma diversidade climática, topográfica e de enormes variações nas precipitações anuais, tanto em latitude, longitude, como em altitude (Junk 2013). 
São AUs interiores, com nível de água flutuante, sujeitas a pulsos de inundações previsíveis, monomodal (inundação que ocorre uma vez ao ano), de longa duração. As de alta amplitude, ao longo de grandes rios, contemplam as AUs amazônicas e as do rio Paraná; e as de baixa amplitude, as grandes AUs savânicas, tais como Pantanal, rio Araguaia (Ilha do Bananal), rio Guaporé e savanas de Roraima (Junk et al. 2014a, 2014b).

As grandes paisagens de AUs savânicas (Figura 1) ocorrem na transição de gradientes entre as áreas inundáveis pelo transbordamento dos rios e/ou as alagadas pelas chuvas, onde a pluviosidade varia entre 1000 a 2000 $\mathrm{mm} \mathrm{a}^{-1}$ (Junk et al. 2014b), e compreendem um mosaico de vegetações hidrófilas, savanas alagáveis e manchas de florestas alagáveis e não alagáveis, todas adaptadas a secas severas e ao impacto do fogo. O manejo dessas paisagens complexas necessita de uma classificação das diferentes unidades hidrológicas e florísticas chamadas macro-habitat (Junk et al. 2015, Nunes da Cunha \& Junk 2017a), para garantir o seu uso sustentável e sua proteção. Essa classificação já foi elaborada para o Pantanal, por Nunes da Cunha \& Junk (2014, 2017b), e para as várzeas e igapós amazônicos, por Junk et al. (2014c, 2015).

O Pantanal é uma AU que pertence à categoria de savanas do tipo periodicamente inundadas; portanto, parte do bioma Cerrado (Nunes da Cunha \& Junk 2011). É a AU savânica mais bem estudada. A sua vegetação é composta de diferentes tipos de savanas naturais (Costa et al. 2010, Ferreira-Jr et al. 2016) e de campos (Rebellato \& Nunes da Cunha 2005, Nunes da Cunha et al. 2007), podendo ser entrecortada por brejos (Nunes da Cunha et al. 2006, Nunes da Cunha \& Junk 2014). Além disso, existem manchas de florestas inundáveis (Damasceno-Jr et al. 2005, Arieira \& Nunes da Cunha 2006) e não inundáveis (Nunes da Cunha et al. 2006, 2007). Algumas são do tipo monodominantes - por exemplo, os paratudais e os canjiqueirais (Nunes da Cunha et al. 2006, Nunes da Cunha \& Junk 2014), de formações de arbustos (arbustais) (Silva et al. 2016, Arruda et al. 2017) e campo de murunduns (Nunes da Cunha et al. 2006, Nunes da Cunha \& Junk 2014). Atualmente descrito com 56 macro-habitat, o Pantanal é a AU mais diversa do Brasil, pois inclui macro-habitat de savanas, de florestas e campos periodicamente inundáveis, além dos terraços aluviais e paleoleque não inundados atualmente (Nunes da Cunha \& Junk 2017b).

As AUs savânicas dos rios Araguaia e Guaporé pertencem aos biomas Cerrado e Amazônia, respectivamente, e apresentam características vegetacionais similares às do Pantanal (Junk et al. 2014b). O Araguaia possui um tipo peculiar de floresta inundável que ocorre com elementos florísticos do Cerrado e Floresta Amazônica, evidenciando seu caráter transicional (Arruda, com. pessoal). Dispõem ainda de florestas abertas monodominantes, os paratudais e os canjiqueirais, as áreas abertas campestres com agrupamento de arbusto e indivíduos arbóreos, conhecidas como campo de murunduns (Marimon \& Lima 2001) e pequenos fragmentos de florestas naturais em terreno côncavo, conhecidos como ipucas ou impucas (Brito et al. 2006, 2007; Barbosa et al. 2011). A vegetação das AUs do rio Guaporé engloba grandes áreas de savanas inundáveis, com diferentes formações herbáceas (campos naturais) e florestas inundáveis (igapó), além de amplos buritizais (Bisaggio et al. 2013). O Instituto Nacional de Ciência e Tecnologia em Áreas Úmidas (INCT - INAU) vem desenvolvendo estudos nas AUs dos rios Araguaia e Guaporé visando à classificação dos seus macro-habitat. Uma grande contribuição para a região do Araguaia foi a determinação da extensão de sua planície de inundação, a partir de técnicas de sensoriamento remoto (Nunes \& Dias 2017) e da consideração sobre a idade dos sedimentos (Irion et al. 2016) para melhor compreensão do funcionamento dos macro-habitat.

Quanto às savanas de Roraima, conhecidas popularmente como lavrados (Barbosa et al. 2007), inseridas no bioma Amazônia, sua vegetação é composta pelos buritizais e campinaranas (Barbosa \& Miranda 2004, Barbosa et al. 2007). Embora muitos trabalhos retratem as savanas de Roraima (Miranda \& Absy 2000, Barbosa \& Fearnside 2005, Barbosa \& Miranda 2004, Barbosa et al. 2005, 2007), poucos as reconhecem como uma área úmida (Morais \& Carvalho 2015, Carvalho et al. 2016). 


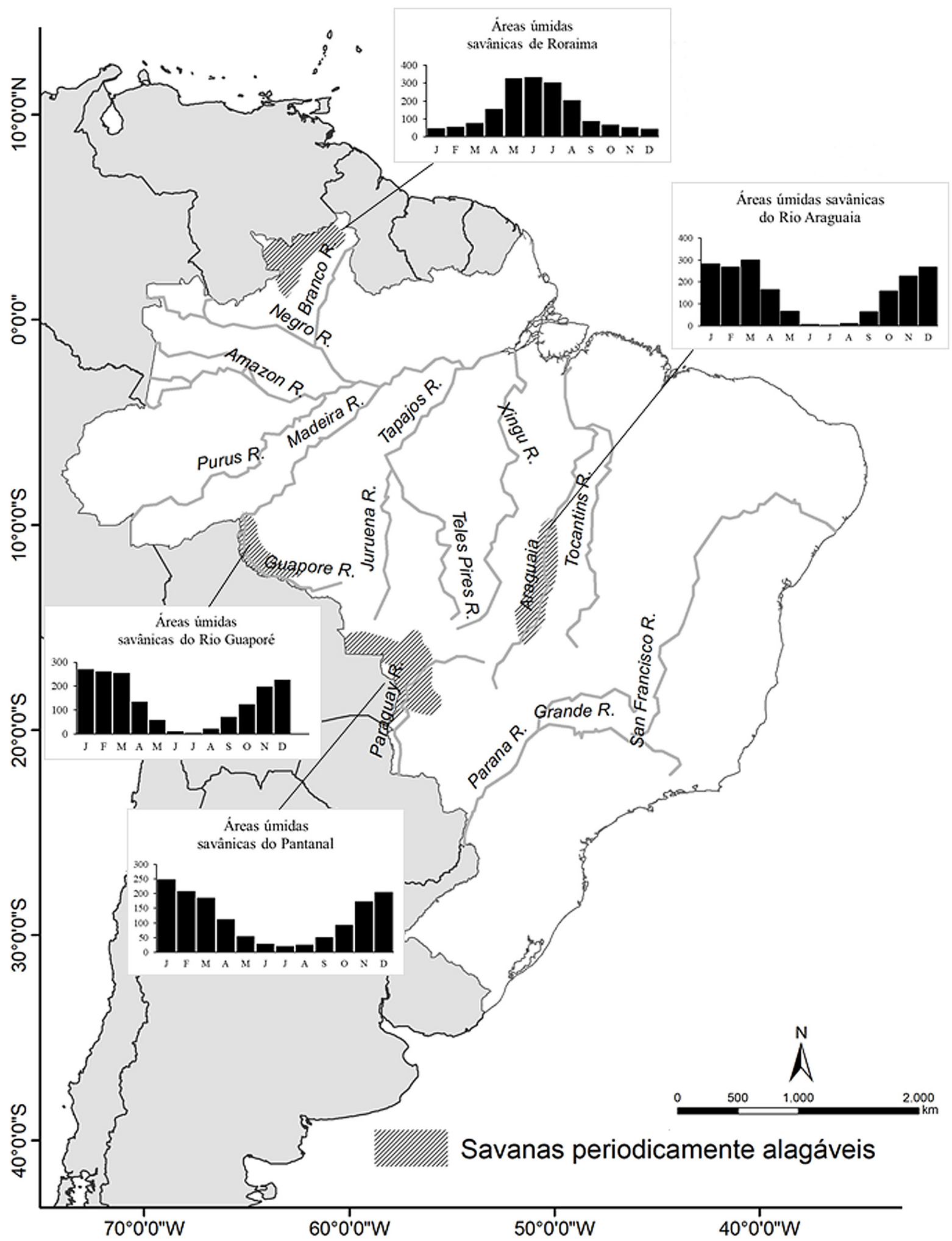

Figura 1 - Mapa das grandes AUs savânicas periodicamente alagadas que ocorrem no Brasil. 
Dos 22 sítios Ramsar estabelecidos no Brasil (MMA 2018), seis estão localizados nas AUs savânicas, sendo três no Pantanal (Parque Nacional do Pantanal Matogrossense, Reserva Particular do Patrimônio Natural SESC Pantanal e Reserva Particular do Patrimônio Natural Fazenda Rio Negro); um na AU do rio Araguaia (Parque Nacional do Araguaia - Ilha do Bananal), um na AU do rio Guaporé (Reserva Biológica do Guaporé) e um nas savanas de Roraima (Parque Nacional de Viruá). Apesar dos esforços para o estabelecimento de políticas de proteção das AUs e criação de sítios Ramsar em todo o mundo, mais de $50 \%$ desses ambientes já foram destruídos ou tiveram sua integridade comprometida (Dugan 1993, Gibbs 2000, Turner et al. 2000, Mitsch \& Gosselink 2008, Gopal 2013).

Para as AUs brasileiras, as principais ameaças são oriundas das diversas atividades econômicas realizadas de modo inconsequente pelos diferentes atores sociais, aliadas à ineficiência do controle das leis ambientais e da equivocada implementação de políticas públicas (Junk et al. 2014b, Junk 2017).

Para as AUs savânicas, o processo de transformação se inicia a partir da expansão das fronteiras agrícolas no bioma Cerrado (Fernandes et al. 2016), onde as principais ameaças são: (1) transformação da paisagem, com destruição de macro-habitat essenciais, através de drenagem $e$ desmatamento para ampliação de cultivos agrícolas de larga escala (soja, arroz e milho) (Barbosa et al. 2005, Alho \& Sabino 2011, Junk et al. 2014b, Fernandes et al. 2016, SEMA 2016, Garcia et al. 2017, Junk 2017, WWF 2017); (2) intensificação da pecuária; (3) modificação da hidrologia (Da Silva \& Girard 2004, Alho \& Sabino 2011, Junk et al. 2014b, Junk 2017); (4) introdução de espécies (Junk 2017) de outros ecossistemas e/ou exóticos, tanto vegetais (capins exóticos, como Urochloa humidicola (Rendle) Morrone \& Zuloaga e Urochloa decumbens (Stapf) R.D.Webster), quanto animais (búfalos, javalis e tucunaré) (Junk et al. 2006, Alho \& Sabino 2011, Bisaggio et al. 2013, Junk 2017); e (5) construção de hidroelétricas (Calheiros et al. 2009, Alho \& Sabino 2011, Junk et al. 2014b, Irigaray et al. 2017, Junk 2017).

Essas savanas têm sido usadas tradicionalmente para pecuária extensiva (Nunes da Cunha et al. 2006, Soares et al. 2009, Hoogesteijn \& Hoogesteijn 2010, Junk \& Nunes da Cunha 2016) e pesca (Resende \& Santos 2002, Almeida \& Resende 2012, Polaz et al. 2014). Além disso, há o uso local da madeira (Carniello et al. 2011), dos frutos e sementes (Bortolotto et al. 2015, Urbanetz et al. 2016, Bortolotto et al. 2017), da seiva e da casca do tronco de árvores (Guarim Neto et al. 2008, Pinto et al. 2017). O ecoturismo, em combinação com as outras atividades, é considerado a melhor opção para o sucesso do manejo sustentável e para a conservação desses ambientes e de sua biodiversidade (Girard \& Vargas 2008, Junk 2017).

\section{Para a Convenção Ramsar, o que quer dizer o termo uso racional?}

O centro filosófico da Convenção Ramsar é a adoção do "Princípio do Uso Racional" das AUs (Ramsar 2010), o qual reconhece a interdependência do homem e o desenvolvimento sustentável dos recursos naturais presente nesses ambientes (Finlayson et al. 2011, Tiéga 2011). A partir do momento em que aderem à Convenção, as partes contratantes comprometem-se a trabalhar para o uso racional de todas as AUs e dos recursos hídricos em seus territórios, através de planos, políticas e legislações locais e nacionais, ações de gestão e educação pública (Ramsar 2010).

A definição atualizada de "uso racional" das AUs foi elaborada considerando-se a missão da Convenção Ramsar, as terminologias utilizadas pela Avaliação Ecossistêmica do Milênio, os conceitos de abordagem ecossistêmica e uso sustentável da Convenção da Diversidade Biológica $e$ a definição de desenvolvimento sustentável adotada pela Comissão de Brundtland. Assim, "O uso racional das AUs é a manutenção de suas características ecológicas, alcançadas através da implementação de abordagens ecossistêmicas, no contexto do desenvolvimento sustentável" (Ramsar 2010). 
O Brasil, como signatário da Convenção Ramsar, deve compatibilizar suas políticas públicas, através do reconhecimento dos múltiplos valores das AUs, alcançando, assim, o uso racional dos seus recursos e contribuindo para o desenvolvimento sustentável (Ramsar 2010).

\section{A aplicação do conceito do uso múltiplo nas áreas úmidas savânicas brasileiras}

No Brasil, AUs savânicas não são contempladas na legislação específica (Junk et al. 2014a, 2014b). Entretanto, algumas dessas áreas são protegidas por diferentes tipos de unidades de conservação, incluindo sítios Ramsar (Junk 2013, Junk et al. 2013). A orientação de seu manejo, uma

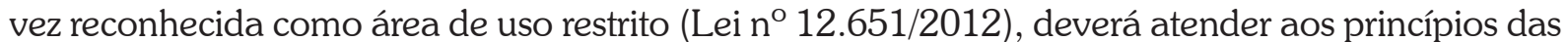
Convenções Ramsar e da Diversidade Biológica através das Metas de Aichi estabelecidas durante a $10^{\text {a }}$ Conferências das Partes $(\mathrm{COP} / \mathrm{CDB})$, voltadas para a redução da perda da biodiversidade em âmbito mundial. Sítios Ramsar são as únicas áreas que possuem convenção internacional própria (Turner et al. 2000). Desde 2011, o Brasil vem desenvolvendo mecanismos para aprimorar e implementar a estratégia e o plano de ação nacional para a biodiversidade.

As AUs oferecem múltiplos serviços ambientais (Turner et al. 2000, Keddy et al. 2009, Junk et al. 2010, Junk et al. 2014a, Adade et al. 2017, Junk 2017), que podem ser divididos em serviços comerciais e não comerciais. No Pantanal Matogrossense, serviços comerciais são, por exemplo, moradia para a população local, pecuária, pesca, ecoturismo e fornecimento de produtos madeireiros e não madeireiros (fibras, plantas medicinais, frutas). Serviços não comerciais são a estocagem periódica da água e sua lenta devolução para os córregos e rios conectados, reduzindo, com isso, as flutuações do nível da água e o perigo de enchentes e secas catastróficas, além da recarga dos aquíferos e do lençol freático, retenção de sedimentos, fornecimento de água limpa, purificação da água poluída, regulação do microclima, manutenção da biodiversidade, recreação (banho, pesca, lazer), estocagem de carbono orgânico, e manutenção de valores culturais (e.g. música, arte, peculiaridades da vida campestre tradicional). Isso mostra que a maioria dos serviços ambientais do Pantanal são não comerciais.

Atualmente há diversos valores econômicos das AUs savânicas reconhecidos, como, por exemplo, pecuária extensiva (Da Silva \& Girard 2004, Soares et al. 2009, Hoogesteijn \& Hoogesteijn 2010, Junk \& Nunes da Cunha 2016), ecoturismo (Zimmermann et al. 2005, Hoogesteijn \& Hoogesteijn 2010, Tortato \& Izzo 2017, Tortato et al. 2017), fitoterápicos (Schmeda-Hirschmann et al. 2014, Dall'Oglio et al. 2015, Santos et al. 2016, Siqueira et al. 2016) e pesca (Resende \& Santos 2002, Mateus et al. 2004, Almeida \& Resende 2012, Polaz et al. 2014, Barletta et al. 2016, Catella et al. 2016).

Cerca de $90 \%$ da área do Pantanal são fazendas de propriedades privadas (Junk 2017), onde a pecuária é a principal atividade econômica. Outra atividade que exerce forte influência na economia do Pantanal é a pesca, nas modalidades profissional, amadora e de subsistência, com cerca de 10,3 mil pescadores cadastrados no Ministério da Pesca e Aquicultura. Essa atividade gera, aproximadamente, $1207^{-1}$ toneladas/ano de pescado (Barletta et al. 2016), movimentando também o turismo de pesca, com mais de 13 mil pescadores esportivos - isso só no estado de Mato Grosso do Sul (Catella et al. 2016).

Diante das demandas das comunidades locais e de regiões adjacentes por produtos (tais como arroz, laticínios, proteína animal - peixe e carne bovina, lenha, carvão, madeira, frutos comestíveis, extratos fitoterápicos etc.) fornecidos pelas AUs (Obiero et al. 2012; Lamsal et al. 2017), devem-se adotar planos de manejo diferenciados. Primeiramente, essas áreas devem ser reconhecidas como AUs, e a sua regulamentação deve ser realizada através de políticas ambientais (Bassi et al. 2014). Em seguida, é necessária uma classificação dos habitat (Wantzen et al. 2008), aliada a um inventário participativo do ecossistema, associado ao conhecimento tradicional e local sobre a biodiversidade e o pagamento dos serviços ambientais (Deepak 2016). Esses aspectos, 
conciliados a planos de manejo baseados no uso múltiplo, atendem ao conceito de uso restrito previsto na Lei $n^{\circ} 12.651 / 2012$, e aos princípios do uso racional, segundo a Convenção Ramsar (handbook 1) (Ramsar 2010).

O uso múltiplo sustentável requer o manejo das áreas úmidas de uma forma equilibrada, mantendo intactos os seus serviços comerciais e não comerciais. Isso significa a otimização do uso de todos os recursos, ao invés da maximização do benefício de um único recurso. Por exemplo, a maximização da produção de carne bovina em áreas úmidas savânicas iria resultar na destruição de muitos macro-habitat florestados para o plantio de pastos exóticos, destruindo os recursos madeireiros, com consequências desastrosas para a biodiversidade (Figura 2). Além disso, a concentração no manejo de um único recurso deixa o sistema econômico muito vulnerável frente a diversas ameaças, e.g. enchentes e secas extremas, doenças de gado, queda de preços etc.

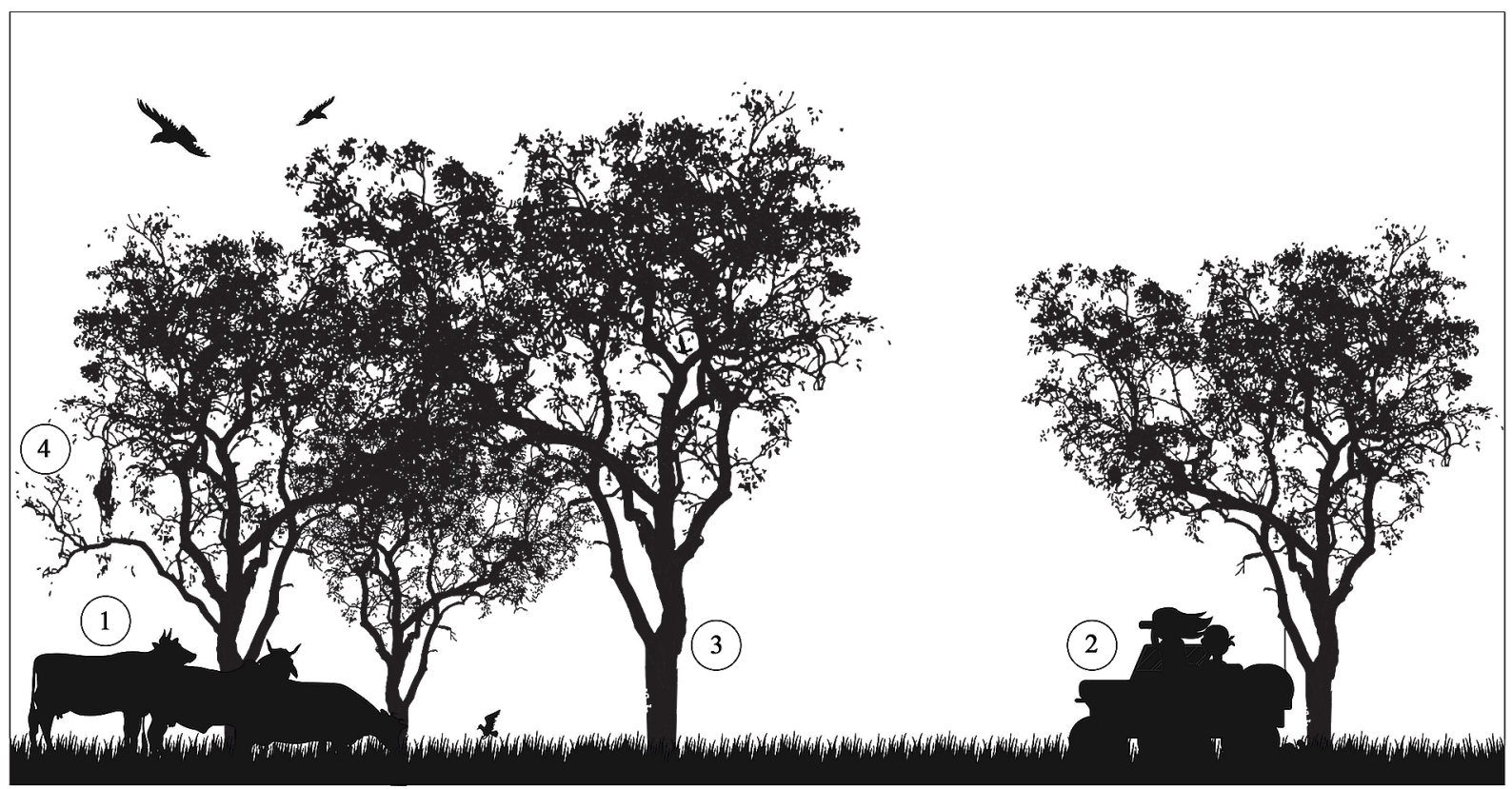

Figura 2 - Os múltiplos usos das savanas de áreas úmidas em: 1) forrageamento; 2) observação de pássaros; 3) recurso madeireiro; e 4) proteção da biodiversidade.

Outra vantagem do manejo de uso múltiplo é o envolvimento de todas as partes interessadas na obtenção de benefícios dos diferentes recursos naturais, diminuindo os conflitos sociais e incentivando a manutenção dos diferentes macro-habitat, inclusive a sua biodiversidade (Sabogal et al. 2013, Nix 2017).

Experiências demonstram o sucesso da aplicação prática do manejo de uso múltiplo em áreas de terra firme, como a colheita manual de produtos florestais no estado do Acre (látex, castanhas do Brasil, após planos de manejo) e a produção diversificada de produtos não madeireiros (couro orgânico, óleos, mel, produtos florestais para uso medicinal e culturas perenes em agricultura de subsistência) nas reservas extrativistas (RESEX), reservas de desenvolvimento sustentável (RDS) e projetos de assentamento nos estados do Pará, Amazonas e Rondônia, tanto em áreas de terra firme, quanto nas AUs (Sabogal et al. 2013). A abordagem de uso múltiplo inclui também espécies lenhosas (uso medicinal, confecção de ferramentas domésticas e agrícolas, brinquedos, objetos para uso doméstico e artesanato), como demonstrado na região semiárida brasileira por Lucena et al. 2007. Embora para comunidades indígenas não seja permitida a comercialização da madeira, o comércio de produtos não madeireiros (óleos, fibras naturais processadas e artesanatos confeccionados a partir de sementes) é permitido e realizado com sucesso (Sabogal et al. 2013). 
Contudo, a atual visão agropecuária brasileira está alterando a estrutura e o funcionamento das AUs em grande escala, principalmente através do desmatamento, do plantio de capins exóticos e da drenagem de áreas pantanosas. Outros impactos negativos resultam da alteração do pulso de inundação por meio de construções de hidroelétricas. Os planos para a construção de hidrovia são uma ameaça contínua para a integridade ecológica do Pantanal Matogrossense (Harris et al. 2005a, 2005b, 2006, Alho 2008, 2011, Junk \& Nunes da Cunha 2012).

A adoção do uso múltiplo pelos proprietários representa uma ferramenta imprescindível na conservação da biodiversidade (proteção dos macro-habitat, garantia da manutenção dos serviços ambientais ecossistêmicos produzidos pelas áreas úmidas) e no aumento dos benefícios econômicos para os proprietários. Assim, o setor privado desempenha um papel indispensável na conservação das áreas úmidas savânicas, obtendo vantagens lucrativas com, por exemplo, o ecoturismo (Hoogesteijn \& Hoogesteijn 2010). Considerando que grande parte das áreas úmidas savânicas são de propriedades privadas, a abordagem de uso múltiplo e o incentivo para criação de reservas particulares do patrimônio natural (RPPN) e outras categorias de unidades de conservação são de grande relevância para proteção e manutenção dessas savanas.

\section{Recomendações para promoção do uso múltiplo para as áreas úmidas savânicas}

O conhecimento sobre as savanas inundáveis deve ser divulgado, para que seu uso seja realizado de forma sustentável e garanta seu aproveitamento por futuras gerações. Propomos as seguintes estratégias e ações para que o uso múltiplo desses macro-habitat seja praticado de maneira sustentável:

- realizar mais pesquisas científicas nas AUs savânicas (Araguaia, Pantanal, Guaporé e savanas de Roraima), pois o conhecimento científico é a base essencial para subsidiar as formas de uso múltiplo, a proteção e sua conservação;

- utilizar a classificação dos macro-habitat para identificar quais são adequados para uso múltiplo;

- mapear os macro-habitat principais em cada AU para quantificar a sua área, determinar a sua presença dentro da respectiva AU e verificar o seu estágio de integridade ecológica;

- valorizar a cadeia produtiva das espécies de plantas e animais usadas;

- incentivar e promover a adoção de pagamento por serviços ambientais, principalmente em áreas de uso múltiplo, por manterem as características ecológicas desses macro-habitat;

- fortalecer e criar unidades de conservação, principalmente onde exista pressão antrópica;

- envolver os atores locais em práticas estratégicas na busca de soluções sustentáveis, pois seus conhecimentos são fundamentais na conservação desses locais;

- identificar as áreas mais ameaçadas dentro das AUs savânicas e estabelecer planos de restauração para esses ambientes;

- estimular campanhas para divulgar a beleza cênica e a importância dessas AUs savânicas para toda a sociedade;

- promover o turismo ecológico dessas AUs e nas unidades de conservação, visando ao uso público como ação educativa e sustentabilidade financeira;

- promover políticas públicas específicas para as AUs savânicas, baseadas no conhecimento científico para sua conservação;

- capacitar gestores tomadores de decisão sobre o uso múltiplo das AUs savânicas e promover uma integração entre tomadores de decisão e comunidade científica; 
- investir em agendas/programas de educação ambiental para a população local, promovendo a valorização das savanas com intuito de diminuir a destruição dessas áreas; $e$

- incentivar a manutenção de valores culturais (e.g. música, arte, peculiaridades da vida campestre tradicional).

\section{Conclusão}

Mudanças de paradigmas sobre o uso das AUs pelos atores que atuam nessas áreas, principalmente as que são de uso restrito, segundo lei federal, são necessárias, para que se possa visualizar as oportunidades que esses ambientes fornecem, sem a necessidade de alterá-los. $\mathrm{O}$ modelo de uso múltiplo deve ser proposto para que sejam criadas alternativas de uso sustentável dessas áreas úmidas savânicas onde a paisagem, a biodiversidade e outros serviços ambientais possam estar associados à atividade econômica principal, como pecuária extensiva, pesca $e$ turismo. O turismo é uma atividades muito explorada em outras savanas do mundo. Todavia, no Brasil essa prática ainda é pouco utilizada, embora as áreas úmidas savânicas brasileiras ofertem uma maior quantidade de atrações turísticas, tais como abundância de animais silvestres, beleza paisagística, pesca, esportiva, pecuária extensiva e valores culturais.

Nesse contexto, as áreas florestadas representam, em áreas úmidas savânicas, macro-habitat de alto valor para a manutenção da biodiversidade e, por isso, deveriam ser protegidas. No conceito do uso múltiplo, a extração de madeira deveria ser restrita para uso local sob controle rígido dos órgãos competentes. A aplicação do uso múltiplo no manejo pode ser a forma mais eficaz de promover a conservação e o uso sustentável da biodiversidade nas AUs savânicas, atendendo aos compromissos assumidos pelos países em relação às metas de Aichi (Convenção sobre a Diversidade Biológica - CDB). Somente com o manejo dessa natureza é que podemos trabalhar em busca de pagamentos pelos serviços ambientais, como proposto pelas comunidades científicas e organizações internacionais, como as Nações Unidas (Pagiola et al. 2005, Farley \& Costanza 2010, Lipper \& Neves 2011).

\section{Referências bibliográficas}

Adade, R.; Nyarko, B.K.; Aheto, D.W. \& Osei, K.N. 2017. Fragmentation of wetlands in the south eastern coastal savanna of Ghana. Regional Studies in Marine Science, 12: 40-48.

Alho, C.J.R. 2008. Biodiversity of the Pantanal: response to seasonal flooding regime and to environmental degradation. Brazilian Journal of Biology, 68: 957-966.

Alho, C.J.R. 2011. Biodiversity of the Pantanal: its magnitude, human occupation, environmental threats and challenges for conservation. Brazilian Journal of Biology, 71: 229-232.

Alho, C.J.R. \& Sabino, J. 2011. A conservation agenda for the Pantanal's biodiversity. Brazilian Journal of Biology, 71: 327-335.

Almeida, I.M. \& Resende, E.K. 2012. Alimentação dos peixes detritívoros da Baía Tuiuiú, Rio Paraguai, Pantanal de Mato Grosso do Sul, Brasil. Boletim de Pesquisa e Desenvolvimento - EMBRAPA. Corumbá, Embrapa Pantanal. Comunicado Técnico 115. 19p.

Arieira, J. \& Nunes da Cunha, C. 2006. Fitossociologia de uma floresta inundável monodominante de Vochysia divergens Pohl (Vochysiaceae), no Pantanal Norte, MT, Brasil. Acta Botanica Brasilica, 20: 569-580.

Arruda, E.C.; Paixão, E.; Pinheiro da Silva, C.M.V. \& Nunes da Cunha, C. 2017. Understory of the seasonallyflooded brevi-semideciduous Vochysia divergens forest, Pantanal, Brazil. In: Soares, M.A. \& Jardim, M.A.G. (orgs.). Natural Resources in Wetlands: from Pantanal to Amazonia. 288p.

Barbosa, R.I. \& Fearnside, P.M. 2005. Fire frequency and area burned in the Roraima savannas of Brazilian Amazonia. Forest Ecology anda Management, 204: 371-384. 
Barbosa, R.I. \& Miranda, I.S. 2004. Fitofisionomias e diversidade vegetal das Savanas de Roraima. In: Barbosa, R.I.; Xaud, H.A.M. \& Souza, J.M.C. (orgs.). Savanas de Roraima: Etnoecologia, Biodiversidade e Potencialidades Agrossilvipastoris. FEMACT. 202p.

Barbosa, R.I.; Nascimento, S.P.; Amorim, P.A.F. \& Silva, R.F. 2005. Notas sobre a composição arbóreoarbustiva de uma fisionomia das savanas de Roraima, Amazônia Brasileira. Acta Botanica Brasilica, 19: 323-329.

Barbosa, R.I.; Campos, C.; Pinto, F. \& Fearnside, P.M. 2007. The "Lavrados" of Roraima: biodiversity and conservation of Brazil's amazonian savannas. Funct Ecosyst Communities, 1: 29-41.

Barbosa, D.C.F.; Marimon, B.S.; Lenza, E.; Marimon-Junior, B.H.; Oliveira, E.A. \& Maracahipes, L. 2011. Estrutura da vegetação lenhosa em dois fragmentos naturais de florestas inundáveis (impucas) no parque Estadual do Araguaia, Mato Grosso. Rev. Árvore, 35: 457-471.

Barletta, M.; Cussa, V.E.; Agostinho, A.A.; Baigún, C.; Okada, E.K.; Catella, A.C.; Fontoura, N.F; Pompeu, P.S.; Jiménez-Segura, L.F;; Batista, V.S.; Lasso, C.A.; Taphorn, D. \& Fabré, N.N. 2016. Fisheries ecology in South American river basins. In: Craig, J.F. (org.). Freshwater Fisheries Ecology, 918p.

Bassi, N.; Kumar, M.D.; Sharma, A. \& Pardha-Saradhi, P. 2014. Status of wetlands in India: A review of extent, ecosystem benefits, threats and management strategies. Journal of Hydroloy: Regional Studies, 2: 1-19.

Bisaggio, E.L.; Alves, S.L.; Costa, C.; Júnior, S.; Henrique, C. \& Rocha, B. 2013. Búfalos Ferais (Bubalus bubalis) em Áreas Protegidas: um estudo de caso na Reserva Biológica do Guaporé, RO. Biodiversidade Brasileira, 3: 243-260.

Bortolotto, I.M.; Mello Amorozo, M.C.; Guarim-Neto, G.; Oldeland, J. \& Damasceno-Junior, G.A. 2015. Knowledge and use of wild edible plants in rural communities along Paraguay River, Pantanal, Brazil. J. Ethnobiol. Ethnomed., 11: 46.

Bortolotto, I.M.; Hiane, P.A.; Ishii, I.H.; Souza, P.R.; Campos, R.P.; Gomes, R.J.B.; Farias, C.S.; Leme, F.M.; Oliveira Arruda, R.C.; Corrêa da Costa, L.B. \& Damasceno-Junior, G.A. 2017. A knowledge network to promote the use and valorization of wild food plants in the Pantanal and Cerrado, Brazil. Reg. Environ. Chang., 17: 1329-1341.

Brasil, 2012. Lei $\mathrm{n}^{\circ}$ 12.651, de 25 de maio de 2012. Dispõe sobre a proteção da vegetação nativa; e dá outras providências. Diário Oficial da República Federativa do Brasil, Brasília, DF, 2012. < http:// www.planalto.gov.br/ccivil_03/_ato2011-2014/2012/lei/L12651compilado.htm> (Acesso em 22/01/2018).

Brito, E.R.; Martins, S.V.; De Oliveira Filho, A.T.; Silva, E. \& Da Silva, A.F. 2006. Phytosociological structure of a natural fragment of floodplain forest in area of irrigated rice cultivation, municipal district of Lagoa da Confusão, Tocantins. Brazil. Rev. Árvore, 30: 829-836.

Brito, C.F.P.; Martins, I.C.M. \& Martins, A.K.E. 2007. Avaliação multitemporal da regeneração dos fragmentos florestais naturais ipucas, Lagoa da Confusão-TO, p.: 2365-2372. In: Simpósio Brasileiro de Sensoriamento Remoto. Florianópolis. Anais. INPE.

Calheiros, D.F.; Arndt, E.; Rodriguez, E.O. \& Silva, M.C. 2009. Influências de usinas hidrelétricas no funcionamento hidroecológico do Pantanal Mato-Grossense: Recomendações. Série Doc. - EMBRAPA Pantanal. 21p.

Carniello, M.A.; Guarim-Neto, G.; Santos Guarim, V.L.M.; Figueiredo, Z.N. \& Amorozo, M.C.M. 2011. Traditional use of vegetation for cattle-raising in the Pantanal on the Brazilian-Bolivian border. In: Junk, W.J.; Da Silva, C.; Nunes da Cunha, C. \& Wantzen, K.M. (orgs.). The Pantanal: Ecology, Biodiversity and Sustainable Management of a Large Neotropical Seasonal Wetland. 870p.

Carvalho, T.M.; Carvalho, C.M. \& Morais, R.P. 2016. Fisiografia da Paisagem e Aspectos Biogeomorfológicos do Lavrado, Roraima, Brasil. Rev. Bras. Geomorfol., 17: 93-107.

Catella, A.C.; Campos, F.L.R. \& Albuquerque, S.P. 2016. Sistema de Controle da Pesca de Mato Grosso do Sul SCPESCA/MS - 22 - 2015. Boletim de Pesquisa e Desenvolvimento - EMBRAPA. EMBRAPA Pantanal. Comunicado Técnico, 131. 56p. 
CBD - Convenção da Diversidade Biológica. 2010. Living in Harmony with Nature Strategic Plan for the Cartagena Protocol on Convention on Biological Diversity.

Costa, C.P.; Nunes da Cunha, C. \& Costa, S.C. 2010. Caracterização da flora e estrutura do estrato arbustivoarbóreo de um cerrado no Pantanal de Poconé, MT. Biota Neotrop., 10: 61-73.

Da Silva, C.J. \& Girard, P. 2004. New challenges in the management of the Brazilian Pantanal and catchment area. Wetl. Ecol. Manag., 12: 553-561.

Dall'Oglio, E.L.; De Sousa, P.T.; Campos, D.C.; Vasconcelos, L.G.; Da Silva, A.C.; Ribeiro, F.; Rodrigues, V. \& Kuhnen, C.A. 2015. Measurement of dielectric properties and microwave-assisted homogeneous acidcatalyzed transesterification in a monomode reactor. J. Phys. Chem, 119: 8971-8980.

Damasceno-Junior, G.A.; Semir, J.; Santos, F.A.M. \& Leitão-Filho, H.F. 2005. Structure, distribution of species and inundation in a riparian forest of Rio Paraguai, Pantanal, Brazil. Flora, 200: 119-135.

Deepak, V. 2016. Development with an ecological perspective focusing on wetlands of Kadamakudy Panchayat, Kochi. Procedia Technol., 24: 1691-1698.

Dugan, P. 1993. Wetlands in Danger: a World Conservation Atlas. Oxford University Press. 187p.

Farley, J. \& Costanza, R. 2010. Payments for ecosystem services: From local to global. Ecol. Econ., 69: 2060-2068.

Fernandes, G.W.; Pedroni, F.; Sanchez, M.; Scariot, A.; Aguiar, L.M.S.; Ferreira, G.; Machado, R.; Ferreira, M.E.; Diniz, S.; Pinheiro, R.; Costa, J.A.S.; Dirzo, R. \& Muniz, F. 2016. Cerrado: em Busca de Soluções Sustentáveis. Vertentes Produções Artísticas. 212p.

Ferreira-Junior, W.; Schaefer, C.E.G.R.; Nunes da Cunha, C.; Duarte, T.; Chieregatto, L.C. \& Carmo, F.M.S. 2016. Flood regime and water table determines tree distribution in a forest-savanna gradient in the Brazilian Pantanal. An. Acad. Bras. Cienc., 88: 1-13.

Finlayson, C.M.; Davidson, N.; Pritchard, D.; Randy Milton, G. \& MacKacy, H. 2011. The ramsar convention and ecosystem-based approaches to the wise use and sustainable development of wetlands. J. Int. Wildl. Law Policy, 14: 176-198.

Garcia, A.S.; Sawakuchi, H.O.; Ferreira, M.E. \& Ballester, M.V.R. 2017. Landscape changes in a neotropical forest-savanna ecotone zone in central Brazil: The role of protected areas in the maintenance of native vegetation. J. Environ. Manage, 187: 16-23.

Gibbs, J.P. 2000. Wetland loss and biodiversity conservation. Conserv. Biol., 14: 314-317.

Girard, P. \& Vargas, I. 2008. Tourism, development and knowledge in Pantanal: Possible dialogues and partnerships. Desenvolvimento e Meio Ambiente, 18: 61-76.

Gopal, B. 2013. Future of wetlands in tropical and subtropical Asia, especially in the face of climate change. Aquat. Sci., 75: 39-61.

Guarim-Neto, G.; Guarim, V.L.M.S.; Macedo, M. \& Nascimento, N.P. 2008. Flora, vegetação e etnobotânica: conservação de recursos vegetais no pantanal. Rev. Gaia, 2: 41-46.

Harris, M.; Tomas, W.; Mourão, G.; Silva, C.J.; Guimarães, E.; Sonoda, F. \& Fachim, E. 2005a. Safeguarding the Pantanal Wetland: threats and conservation initiatives. Conservation Biology, 19: 714-720.

Harris, M.; Tomas, W.; Mourao, G.; Silva, C.J; Sonoda, F. \& Fachim, E. 2005b. Desafios para proteger o Pantanal brasileiro: ameaças e iniciativas em conservação. Megadiversidade, 1: 156-164.

Harris, M.B.; Arcângelo, C.; Pinto, E.C.T.; Camargo, G.; Ramos Neto, M.B. \& Silva, S.M. 2006. Estimated loss of natural cover in upper Paraguay River basin and the Brazilian Pantanal. Nat. Conserv., 4: 164-179.

Hoogesteijn, A. \& Hoogesteijn, R. 2010. Cattle ranching and biodiversity conservation as Allies in south America's flooded savannas. Gt. Plains Res., 20: 37-50. 
Irigaray, C.T.J.H.; Da Silva, C.J.; Nunes da Cunha, C.; Calheiros, D.F.; Girard, P. \& Junk, W.J. 2017. Contribuição técnico-científica ao aprimoramento do marco regulatório visando à proteção do Pantanal Mato-grossense. In: Irigaray, C.T.J.H.; Braun, A. \& Irigaray, M. (orgs.). Pantanal Legal: a Tutela Jurídica das Áreas Úmidas e do Pantanal Matogrossense. 152p.

Irion, G.; Nunes, G.M.; Nunes da Cunha, C.; Arruda, E.C.; Tambelini, M.S.; Dias, A.P.; Morais, J.O. \& Junk, W.J. 2016. Araguaia river floodplain: Size, age, and mineral composition of a large tropical savanna wetland. Wetlands, 36: 945-956.

Junk, W.J.; Bayley, P.B. \& Sparks, R.E. 1989. The flood pulse concept in river-floodplain systems. Can. Spec. Publ. Fish. Aquat. Sci., 106: 110-127.

Junk, W.J.; Nunes da Cunha, C.; Wantzen, K.M.; Petermann, P.; Strüssmann, C.; Marques, M.I. \& Adis, J. 2006. Biodiversity and its conservation in the Pantanal of Mato Grosso, Brazil. Aquat. Sci., 68: 278-309.

Junk, W.J.; Piedade, M.T.F.; Schöngart, J.; Wittmann, F. \& Parolin, P. 2010. Amazonian Floodplain Forests: Ecophysiology, Biodiversity and Sustainable Management. Springer Dordrecht Heidelberg. 634p.

Junk, W.J. \& Nunes da Cunha, C. 2012. Pasture clearing from invasive woody plants in the Pantanal: a tool for sustainable management or environmental destruction? Wetl. Ecol. Manag., 20: 111-122.

Junk, W.J. 2013. Current state of knowledge regarding South America wetlands and their future under global climate change. Aquat. Sci., 75: 113-131.

Junk, W.J.; An, S.; Finlayson, C.M.; Gopal, B.; Kvet, J.; Mitchell, S.A.; Mitsch, W.J. \& Robarts, R.D. 2013. Current state of knowledge regarding the world's wetlands and their future under global climate change: a synthesis. Aquat. Sci., 75: 151-167.

Junk, W.J.; Piedade, M.T.F.; Lourival, R.; Wittmann, F; Kandus, P.; Lacerda, L.D.; Bozelli, R.L.; Esteves, F.A.; Nunes da Cunha, C.; Maltchik, L.; Schöngart, J.; Schaeffer-Novelli, Y. \& Agostinho, A.A. 2014 a. Brazilian wetlands: their definition, delineation, and classification for research, sustainable management, and protection. Aquat. Conserv. Mar. Freshw. Ecosyst., 24: 5-22.

Junk, W.J.; Piedade, M.T.F.; Lourival, R.; Wittmann, F.; Kandus, P.; Lacerda, L.D.; Bozelli, R.L.; Esteves, F.A.; Nunes da Cunha, C.; Maltchik, L.; Schöngart, J.; Schaeffer-Novelli, Y.; Agostinho, A.A.; Nóbrega, R.L.B. \& Camargo, E. 2014b. Definição e Classificação das Áreas Úmidas (AUs) Brasileiras: Base Científica para uma Nova Política de Proteção e Manejo Sustentável. In: Nunes da Cunha, C.; Piedade, M.T.F. \& Junk, W.J. (orgs.). Classificação e Delineamento das Áreas Úmidas Brasileiras e de seus Macrohabitats. EdUFMT. 156p.

Junk, W.J.; Piedade, M.T.F; Schöngart, J. \& Wittmann, F. 2014c. A Classificação dos Macrohabitats das Várzeas Amazônicas. In: Nunes da Cunha, C.; Piedade M.T.F. \& Junk, W.J. (orgs.): Classificação e Delineamento das Áreas Úmidas Brasileiras e de seus Macrohabitats. EdUFMT. 156p.

Junk, W.J.; Wittmann, F.; Schöngart, J. \& Piedade, M.T.F. 2015: A classification of the major habitats of Amazonian black-water river floodplains and a comparison with their white-water counterparts. Wetlands Ecology and Management, 23(4): 677-693.

Junk, W.J. \& Nunes da Cunha, C. 2016. The Pantanal: A Brief Review of its Ecology, Biodiversity, and Protection Status. In: Finlayson, C.M.; Milton, R.; Prentice, C. \& Davidson, N. (orgs.). The Wetland Book II: Distribution, Description and Conservation. Springer. 800p.

Junk, W.J. 2017. Ecoturismo: uma opção de manejo sustentável para o Pantanal? In: Irigaray, C.T.J.H.; Braun, A. \& Irigaray, M. (orgs.). Pantanal Legal: a Tutela Jurídica das Áreas Úmidas e do Pantanal Matogrossense. 152p.

Keddy, P.A.; Fraser, L.H.; Solomeshch, A.I.; Junk, W.J.; Campbell, D.R.; Arroyo, M.T.K. \& Alho, C.J.R. 2009. Wet and Wonderful: The World's Largest Wetlands Are Conservation Priorities. Bioscience, 59: 39-51.

Lamsal, P.; Atreya, K.; Pant, K.P. \& Kumar, L. 2017. Wetland Science. In: Prusty, B.A.K.; Chandra, R. \& Azeez, P.A. (orgs.). Wetland Science: Perspective from South Asia. 586p. 
Lipper, L. \& Neves, B. 2011. Payments for environmental services. What role in sustainable agricultural development? ESA working paper, 11-20, 21. Food and Agriculture Organization of the United Nations.

Lucena, R.F.P; Albuquerque, U.P.; Monteiro, J.M.; Almeida, C.D.F.C.B.R.; Florentino, A.T.N. \& Ferraz, J.S.F. 2007. Useful plants of the semi-arid northeastern region of Brazil - a look at their conservation and sustainable use. Environ. Monit. Assess., 125: 281-290.

Marimon, B.S. \& Lima, E.S. 2001. Caracterização fitofisionômica e levantamento florístico preliminar no Pantanal dos Rios Mortes-Araguaia, Cocalinho, Mato Grosso, Brasil. Acta Bot. Brasilica, 15: 213-229.

Mateus, L.A.F.; Penha, J.M.F. \& Petrere, M. 2004. Fishing resources in the rio Cuiabá basin, Pantanal do Mato Grosso, Brazil. Neotrop. Ichthyol., 2: 217-227.

Ministério do Meio Ambiente (MMA). Sítios Ramsar. Disponível em: <http://www.mma.gov.br/areasprotegidas/instrumentos-de-gestao/s\%C3\%ADtios-ramsar > (Acesso em janeiro de 2018).

Miranda, I. \& Absy, M. 2000. Fisionomia das savanas de Roraima, Brasil. Acta Amaz., 30: 423-440.

Mitsch, W.J. \& Gosselink, J.G. 2008. Wetlands. John Wiley e Sons Inc., Hoboken. 582p.

Morais, R.P. \& Carvalho, T.M. 2015. Aspectos dinâmicos da paisagem do lavrado, Nordeste de Roraima. Geociências, 34: 55-68.

Nix, S. 2017. "Multiple-use Management" < https://www.thoughtco.com/multiple-use-1341734>. (Acesso em janeiro de 2018).

Nunes, G.M. \& Dias, A.P. 2017. Delimitação e mapeamento das áreas úmidas em escala regional. In: Nunes da Cunha, C.; Arruda, E.C. \& Junk, W.J. (orgs.). Marcos Referenciais e Gestão de Outras Áreas Úmidas Marcos Referenciais e Gestão de Outras Áreas Úmidas. 156p.

Nunes da Cunha, C.; Rawiel, C.P.; Wantzen, K.M.; Junk, W.J. \& Lemes do Prado, A. 2006. Mapping and characterization of vegetation units by means of Landsat imagery and management recommendations for the Pantanal of Mato Grosso (Brazil), north of Poconé. Amazoniana, 19: 1-32.

Nunes da Cunha, C.; Junk, W.J. \& Leitão Filho, H.D.F. 2007. Woody vegetation in the Pantanal of Mato Grosso, Brazil: a preliminary typology. Amazoniana, 19: 159-184.

Nunes da Cunha, C. \& Junk, W. 2011. A preliminary classification of habitats of the Pantanal of Mato Grosso and Mato Grosso do Sul, and its relation to national and international wetland, In: Junk, W.J.; Da Silva, C.J.; Nunes da Cunha, C. \& Wantzen, K.M. (orgs.). The Pantanal: Ecology, Biodiversity and Sustainable Management of a Large Neotropical Seasonal Wetland. Pensoft. 870p.

Nunes da Cunha, C. \& Junk, W.J. 2014. A classificação dos macrohabitats do pantanal Mato-grossense In: Nunes da Cunha, C.; Piedade, M.T.F. \& Junk, W.J. (orgs.). Classificação e Delineamento das Áreas Úmidas Brasileiras e de seus Macrohabitats. EdUFMT. 156p.

Nunes da Cunha, C. \& Junk, W.J. 2017a. Classificação de áreas úmidas e aplicação em nível estadual. In: Nunes da Cunha, C.; Arruda, E.C. \& Junk, W.J. (orgs.). Marcos Referenciais para a Lei Federal do Pantanal e Gestão de Outras Áreas Úmidas. EdUFMT. 156p.

Nunes da Cunha, C. \& Junk, W.J. 2017b. Classificação dos macrohabitats do Pantanal mato-grossense para fins de gestão. In: Nunes da Cunha, C.; Arruda, E.C. \& Junk, W.J. (orgs.). Marcos Referenciais e Gestão de Outras Áreas Úmidas. EdUFMT. 156p.

Obiero, K.O.; Wa'Munga, P.O.; Raburu, P.O. \& Okeyo-Owuor, J.B. 2012. The people of Nyando Wetland: socioeconomics, gender and cultural issues. In: Raburu, P.O.; Okeyo-Owuor, J.B. \& Kwena, F. (orgs.). Community Based Approach to the Management of Nyando Wetland, Lake Victoria Basin, Kenya. 157p.

Pagiola, S.; Arcenas, A. \& Platais, G. 2005. Can payments for environmental services help reduce poverty? An exploration of the issues and the evidence to date from Latin America. World Dev, 33: 237-253. https:// doi.org/10.1016/j.worlddev.2004.07.011 
Pinto, J.S.; Oliveira, A.K.M.; Fernandes, V. \& Matias, R. 2017. Etnobotânica e a cultura popular no uso de plantas em assentamentos na borda sul do Pantanal Sul-Mato-Grossense. Biosci. J., 33: 193-203.

Polaz, C.N.M.; Melo, B.F.; Britzke, R.; Resende, E.K.; Arruda Machado, F.; Lima, J.A.F. \& Petrere, M. 2014. Fishes from the Parque Nacional do Pantanal Matogrossense, upper Paraguai River basin, Brazil. Check List, 10: 122-130.

Ramsar. 2010. Wise use of wetlands: concepts and approaches for the wise use of wetlands, Ramsar Handbooks, 4th edition. 60p.

Rebellato, L. \& Nunes da Cunha, C. 2005. Efeito do "fluxo sazonal mínimo da inundação" sobre a composição e estrutura de um campo inundável no Pantanal de Poconé. Acta Bot. Brasilica, 19: 789-799.

Resende, E.K. \& Santos, D.C. 2002. Diagnóstico da pesca e aspectos da biologia reprodutiva dos peixes da bacia hidrográfica do Rio Taquari, Mato Grosso do Sul, Brasil. Boletim de pesquisa e desenvolvimento - EMBRAPA. EMBRAPA Pantanal. Comunicado Técnico. 25. 21p.

Sabogal, C.; Guariguata, M.; Broadhead, J.; Lescuyer, G.; Savilaakso, S.; Essoungou, J.N. \& Sist, P. 2013. Multiple-use forest management in the humid tropics: opportunities and challenges for sustainable forest management. FAO Forestry Paper 2013, $\mathrm{n}^{\circ} 173$. Food and Agriculture Organization of the United Nations, and Bogor, Indonesia, Center for International Forestry Research. 118p.

Santos, C.H.C.; Borges, I.P.; Da Silva, V.C.; de Sousa, P.T.; Kawashita, N.H.; Baviera, A.M. \& Carvalho, M.G. 2016. A new dammarane saponin and other triterpenoids from Siolmatra brasiliensis and evaluation of the antidiabetic activity of its extract. Pharm. Biol., 54: 1539-1547.

Schmeda-Hirschmann, G.; Quispe, C.; Theoduloz, C.; Sousa-Junior.; P.T. \& Parizotto, C. 2014. Antiproliferative activity and new argininyl bufadienolide esters from the "cururú" toad Rhinella (Bufo) schneideri. J. Ethnopharmacol, 155: 1076-1085.

Secretaria de Estado de Meio Ambiente de Mato Grosso (SEMA - MT). 2016. Relatório Áreas Úmidas do Estado de Mato Grosso. Relatório Técnico. 24p.

Silva, F.H.B.; Arieira, J.; Parolin, P.; Nunes da Cunha, C. \& Junk, W.J. 2016. Shrub encroachment influences herbaceous communities in flooded grasslands of a neotropical savanna wetland. Appl. Veg. Sci., 19: 391-400.

Siqueira, J.T.; Batistela, E.; Pereira, M.P.; Silva, V.C.; Sousa Junior, P.T.; Andrade, C.M.B.; Kawashita, N.H.; Bertolini, G.L. \& Baviera, A.M. 2016. Combretum lanceolatum flowers ethanol extract inhibits hepatic gluconeogenesis: an in vivo mechanism study. Pharm. Biol., 54: 1671-1679.

Soares, J.J.; Oliveira, A.K.M. \& De Oliveira, A.K. 2009. The "Paratudal" at the Pantanal de Miranda Corumbá-Ms, Brazil. Rev. Árvore, 33: 339-347.

Tiéga, A. 2011. Ramsar convention on wetlands: 40 years of biodiversity conservation and wise use. J. Int. Wildl. Law Policy, 14: 173-175.

Tortato, F.R. \& Izzo, T.J. 2017. Advances and barriers to the development of jaguar-tourism in the Brazilian Pantanal. Perspect. Ecol. Conserv., 15: 61-63.

Tortato, F.R.; Izzo, T.J.; Hoogesteijn, R. \& Peres, C.A. 2017. The numbers of the beast: Valuation of jaguar (Panthera onca) tourism and cattle depredation in the Brazilian Pantanal. Glob. Ecol. Conserv., 11: 106-114.

Turner, R.K.; van den Bergh, J.C.J.M.; Barendregt, A.; van der Straaten, J.; Maltby, E. \& van Ierland, E.C. 2000. Ecological-economic analysis of wetlands: Scientific integration for management and policy. Ecol. Econ., 35: 7-23.

Urbanetz, C.; Jorge, M.H.A.; Marconcini, J.M. \& Galvani, F. 2016. Protocolos de superação de dormência física e condicionamento de sementes de bocaiuva para pequenos produtores. Boletim de pesquisa e desenvolvimento - EMBRAPA. EMBRAPA Pantanal. Comunicado Técnico. 143. 14p. 
Wantzen, K.M.; Nunes da Cunha, C.; Junk, W.J.; Girard, P.; Rossetto, O.C.; Penha, J.M.; Couto, E.G.; Becker, M.; Priante, G.; Tomas, W.M.; Santos, S.A.; Marta, J.; Domingos, I.; Sonoda, F.; Curvo, M. \& Callil, C. 2008. Towards a sustainable management concept for ecosystem services of the Pantanal wetland. Ecohydrol. Hydrobiol., 8: 115-138.

World Wide Fund for Nature (WWF - Brasil), Universidade Católica Dom Bosco (UCDB), Fundação Tuiuiú. 2017. Monitoramento da Cobertura Vegetal e Uso do Solo da Bacia do Alto Paraguai - 2016. Relatório Técnico. 33p.

Zimmermann, A.; Walpole, M.J. \& Leader-Williams, N. 2005. Cattle ranchers' attitudes to conflicts with jaguar Panthera onca in the Pantanal of Brazil. Oryx, 39: 406-41.

Biodiversidade Brasileira - BioBrasil.

Número temático: Diagnóstico e manejo de áreas úmidas em áreas protegidas

$$
\text { n. 2, } 2019
$$

http://www.icmbio.gov.br/revistaeletronica/index.php/BioBR

Biodiversidade Brasileira é uma publicação eletrônica científica do Instituto Chico Mendes de Conservação da Biodiversidade (ICMBio) que tem como objetivo fomentar a discussão e a disseminação de experiências em conservação e manejo, com foco em unidades de conservação e espécies ameaçadas.

ISSN: 2236-2886 